\title{
Ueber den Reflex in der Umgebung der Macula lutea.
}

\author{
Von \\ Dr. Brecht in Berlin.
}

Hierzu Tafel I und II.

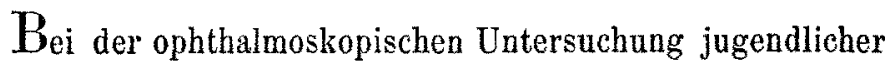
Individuen seben wir bekanntlich oft einen silberglänzenden Reflex, der die Macula lutea umgiebt. Liebreich*) schildert denselben zuerst folgender Weise: "Untersucht man den dunkelpigmentirten Augenhintergrund eines jugendlichen Individuums im umgekehrten Bilde, während man mit dem Spiegel ganz leichte Bewegungen macht, so umkreist der grauliche Schimmer, der den Nervenfasern (welche im Bereich der Macula lutea bekanntlich nicht in continuirlicher Schicht vorhanden sind) seinen Ursprung verdankt, einen rundlichen oder ovalen scharf abgegrenzten Fleck: dieser Fleck, etwas grösser als die Papille ist glanzlos und hinter ihm die Chorioidea etwas dunkler promentirt. In seinem Centrum bemerkt man ein kleines helles Pünktchen (die Mitte der Fovea

*) Graefe's Archiv IV. 2, pag. 301.

v. Graefe's Archir für Ophthalmologie, XXI. 2. 
centralis), umgeben von einem rostfarbenen Hofe, der nach der Peripherie schnell an Deutlichkeit abnimmt". Schirmer*) sagt von dem Phänomen: „Bei allen jugendlichen Individuen erkenne ich die Macula lutea zuerst an einem sie umkreisenden querovalen, hellglänzenden Reif von der Dicke einer Retinalarterie erster Grösse. Der innere Rand dieses Reifens ist scharf, der äussere aber mit kurzen, dichten und feinen Strahlen versehn". Aehnlich sagt Mauthner:**), Dies Queroval besteht in einer silberglänzenden Linie, welche nach innen zu einen vollkommen scharfen Rand besitzt; nach aussen auch gut conturirt sein kann, häufig aber vou ausstrahlenden Lichtbüscheln gekrönt ist".

Ich möchte nach eigener Beobachtung das Phänomen in folgender Weise beschreiben: Der silberglänzende Reflex umgiebt in Kinderaugen die querovale Stelle der Macula lutea, welche übrigens unter gewissen Verhältvissen stellenweis selbst glänzen kann, freilich schwächer als die Umgebung. Immerhin ist die innere Grenze des Reflexes scharf ausgepragt; die äussere dagegen in der Mehrzahl der Fälle nicht, sondern der Glanz verbreitet sich, allmälig schwächer werdend, über die ganze polare Zone und verliert sich allmälig. Oft sieht man freilich auch eine scharfe äussere Begrenzung des Glanzes, sodass ein schmaler glänzender Reif entsteht, welcher kleinere oder grössere Strecken oder die ganze Peri. pherie der Macula lutea einnimmt, aber dann tritt der Reflex, wenn schon etwas schwächer, sehr nahe daneben wieder auf, um sich noch, oft von Gefässen unterbrochen, über die benachbarte Zone auszubreiten. Ein völliges Fehlen dieses letztern flächenhaften Reflexes, so dass also nur der glänzende Reif ubrig bleibt, habe ich nur bei ältern,

*) Graefe's Archiv X, 1, pag. 148.

**) Lehrbuch der Ophthalmoskopie, pag. 314. 
nicht mehr ganz kindlichen Individuen beobachtet. Und noch möchte ich eine Bemerkung erwähnen, die gewiss auch viele Andere gemacht haben, die ich aber nirgends beschrieben finde. Wenn ich das Phänomen in einem Falle recht deatlich wahrgenommen hatte, und nun, um es noch bequemer studiren zu können, das Auge atropinisirte, so fand ich jedesmal zu meiner Verwunderung die Erscheinnng bedeutend geschwächt, wo nicht aufgehoben. Die Erklärung dieses Yorganges werde ich unten zu geben versuchen. Schweigger*) fügt der von Liebreich gegebenen Begründung des Phänomens (dass nämlich der Mangel der zusammenhängenden Nervenfasersehicht den Mangel des Glanzes an der Macula lutea bedinge) noch ein Moment hinzu: Es fehlt in der Macula lutea der in allen ubrigen Stellen der Retina sehr innige Zusammenhang der innern Enden der Radiärfasern mit der Membrana limitans interna, respective es fehlen hier die verbreiterten mit der Membrana limitans verschmelzenden Enden der Radiärfasern selbst, was jedenfalls dazu beitragen muss, den Spiegelglanz der innern Netzhautfläche in der Gegend der Macula lutea zu verringern.

Ueber beide Erklärungen urtheilt Mauthner:**) „Sie mögen uns den Mangel des Glanzes der Macula Jutea erklären, aber sie erklären uns vicht das Phänomen der leuchtenden Fillipse".

In der That müsste ja danach auch der ganze Augenhintergrund mit alleiniger Ausnahme der Macula lutea glänzen, während wir den Reflex doch nur in der Gegend des hintern Pols, in der Umgebung der Macula lutea als schmalen Reif oder mehr weniger breite Zone bemerken.

*) Vorlesungen über den Gebrauch des Augenspiegels, pag. 69. **) loc. cit. pag. 316 . 
Bei der otoskopischen Untersuchung des Trommelfells sieht man in dessen unterer Hälfte einen dreieckigen Reflex, den man sich auch aus einer präsumirten Verdickung der Membran erklärte. Da erwähnte Helm. holt $z^{*}$ ) „dass das Trommelfell nicht in einer einzigen Ebene liege, sondern seine Mitte oder Nabel durch den daran befestigten Hammergriff stark nach innen gezogen. sei in Gestalt eines Trichters, dessen Meridiane gegen seine Höhlung convex sind. Die anatomische Anordnung ist nun der Art, dass die obere Hälfte des Trommelfells fast in gleiche Richtung mit der obern Wand des Gehörgangs zu liegen kommt, und die untere fast senkrecht: auf der Axe dieses Ganges steht. Die Folge davon ist, dass die letztere das von aussen in der Axe des Gehörganges in das $\mathrm{Ohr}$ geworfene Licht wieder gegen den Ausgang des Gehörganges zurückwirft und deshalb als eine dreieckige glänzende Stelle erscheint."

Nach Analogie dieser Erklärung kam ich auf dem Gedanken, dass man sich das oben beschriebene ophthalmoskopische Phänomen auf ähnliche Weise erklären könnte, wenn man an Stelle der Macula lutea eine vielleicht durch das Fehlen der Nervenfaserschicht bedingte Verdünnung der Retina annehme, eine wenn auch sehr geringe Niveauversehiedenhcit der Oberfläehe, eine runde oder querovale Delle. Da der hintere Knotenpunkt und der Mittelpunkt des Auges nicht zusammenfallen, sondern um etwa $4,2 \mathrm{Mm}$. von einander entfernt sind, so treffen nur Strablen, die in der Axe des Auges einfallen, die Tangente ihres Berührungspunktes in der Netzhaut rechtwinkelig. Nur solche werden, sofern sie überhaupt von der Membrana limitans interna gespiegelt werden, in sich selbst zurückgeworfen und kommen dınn natür-

*) Die Mechanik der Gehörinöche'chen. Pflüger's Archiv für Physiologie, I, Jahrgang, pag. 14. 
lich in das beobachtende Auge zurück. Strahlen, die einen Punkt von der Excentricität z. B. der Papille in der Netzhaut treffen, wie a b (in Figur 1)*) bilden mit dem Einfallsloth den Winkel a b c und werden in einem dem letztern gleichen Winkel 0 b c nach der andern Seite reflectirt, in der Richtung b c, verlassen also die Pupille schon nicht mehr. Indess sind die den hintern Pol treffenden Strahlen nicht die einzigen, die wieder durch die Pupille zurückgeworfen werden und so in das Auge des Beobachters gelangen können.

Da nämlich die Pupille nicht ein Punkt ist, sondern eine messbare Ausdehnung hat, so können auch Strahlen, die in einen nicht zu grossen Winkel zur Axe in das Auge einfallen und die Retina in einem nicht $z a$ weit vom hinteren Pole entfernten Punkt treffen, so reflectirt werden, dass sie aus der Pupille zurückgeworfen werden. Der Strahl d e würde in e f reflectirt die Pupille passiren und zwar würde e der äusserste Punkt sein, der Richtungsstrahlen so reflcctirte; dass sie wieder die Pupille träfen und e g würde der Durchmesser des Kreises sein, der bei der Augenspiegelbeleuchtung dem Beobachter ein regelmässiges Reflectiren zeigte; weiter excentrisch gelegene Stellen würden frei von dem regelmässigen Reflex nur das diffuse Licht zur Pupille senden, aus dem nachher das ophthalmoskopische Bild construirt wird.

Danach würden wir nun aber die ganze pólare Zone, wie ich sie nennen will, in der Ausdehnung e $g$ in dem besprochenen Silberglanze sehn, was doch bekanntlich nicht der Fall ist. Denken wir uns jetzt, dass in der Gegend des hintern Pols (an Stelle der Macula

*) Die Figur hat einen Vertikaldurchmesser von $2^{\prime \prime}$, analog dem unten geschilderten künstlichen Auge, ist also etwa 2,5 Mal so gross als das menschliche Auge. Dem entsprechend sind auch die übrigen Verhältnisse gewählt (mit Ausnahme der Iris). 
latea) die innere Netzhautoberfläche eine etwas stärkere Krümmung besitze, wie sie durch das allmähliche Aufhören der Nervenfaserschicht bedingt sein könnte, so leuchtet von selbst ein, dass Richtungsstrahlen, die auf diese mebr concaven Wandungen fallen, in viel grösserem Winkel abgeleitet werden und die Pupille nicht mehr erreichen.

Der Strahl $h \mathrm{f}$ (in Figur II) trifft einen Punkt der (sehr übertrieben concaven) Delle $t \gamma$, welche ihrerseits ganz innerhalb des Kreises e g liegt; aber der Strahl wird jetzt in $f 1$ reflectirt und trifft die Pupille nicht mehr. Nur die den Polpunkt und seine nächste Umgebung, deren Tangenten ja fast rechtwinkelig zur Axo liegen, treffenden Strahlen würden in die Pupille zurückgeworfen, wenn nicht hier sich die stark concav gekrümmte Fovea centralis befände. Der Pol dieser letztern reflectirt nun wirklich sichtbar für den Beobachter, oder besser: die Fovea centr. liefert als Hollspiegel ein physisehes Flammenbildchen in ihrem Brennpunkt, was wir bekanntlich als leuchtenden Punkt oder Stern beim Ophthalmoskopiren beobachten.

Es erhellt aus dem Obigen, dass die Oeffnung der glänzenden polaren Zone, resp. die Breite des glänzenden Ringes direct abhängig ist von dem Durchmesser der Pupille.

Die erstere ist gleichsam ein Bild der letzteren, und man sieht in geeigneten Fällen auch sehr deutlich, dass sich die glänzende Zone mit einer Kreislinie gegen den nicht spiegelnden aber noch erleuchteten Augenhintergrund absetzt.

Bei enger Pupille wird die glänzende Zone schmaler sein, als wenn sie, trotzdem das Licht auf die Macula. lutea auffällt, leidlich gross bleibt, wie bei manchen Kinderaugen; und bei der sehr engen Pupille etwas älterer Individuen wird sie gar nicht mehr als solche 
sichtbar werden. Es wird hier nur der schmale Reif ubrig bleiben: einmal wegen der Enge der Pupille und ferner weil in späteren Jahren mit der abnehmenden Durchsichtigkeit der Membranen und der brechenden Medien der Reflex nur da sichtbar wird, wo die Bedingungen zu seinem Entstehen noch die besten sind. Dass dies aber in der nächsten Nähe der Macula lutea der Fall ist, werde ich unten darzuthun suchen.

Doch ist bisher nur auf die Richtungsstrahlen Bezug genommen, während man hier, wo es sich nicht um die Construction eines Bildes, sonilern nur um Reflex-, um Glanzerscheinungen handelt, auch die Randstrahlen, welche nicht durch den Knotenpunkt gehn, berücksichtigen muss.

Der Gang der Strahlen beim Ophthalmoskopiren ist der: Went die Beleuchtungsflamme sich in $15^{\prime \prime}$ befindet, so würue ein Spiegel von $6^{\prime \prime}$ Brennweite in $10^{\prime \prime}$ ein Flammenbild entwerfen; wenn wir nun die Linse $(+1 / 2)$ in $7 \frac{1}{2}{ }^{\prime \prime}$ Entfernung von unserem Auge und in fast $2^{\prime \prime}$ von der Cornea des beobachteten Auges halten, so bildet sich das Flammenbildchen $A$ (Fig. II a) in $0,9^{\prime \prime}$ oder $23,5 \mathrm{Mm}$. hinter der Linse und in etwa 20,5 vor der Hornhant des beobachteten Anges. Es kann hier durch leichte Drehungen des Spiegels mehr zur Seite, wie auch nach oben und unten vor der I'upille verschoben werden. Wenn man die Randstrahien nicht ignorirt, so bezeichnen in Figur II a ${ }^{*}$ ) die Punkte $r$ und $s$ die Grenzen des Reflexes: Der Randstrahl t $r$ triflt die Retina rechtwinklig in $r$, wird also in sich selbst zurückgeworfen und verlässt die Pupille; alle von weiter unten kommenden Strahlen, werden nach weiter unten reflectirt.

*) Die Pupille ist hier nichs za $7 \mathrm{Mm}$ wie in dem Phantom, sondern zu 8,3 , wie man sie durch die Hornhautvergrösserung in Phantom sieht, angenommen. 
Ebenso verhält es sich mit p s; alle von weiter oben kommenden Strahlen werden weiter nach oben reflectirt als $p$, verlassen also die Pupille nicht mehr, was aber die nicht durch den Mittelpunkt o gehenden Strahlen betrifft, so bilden pf und tf die äussersten Grenzen: tf wird nach fp reflectirt und was von weiter unten als tf kommt, würde auch weiter nach oben als $p$ reflectirt. Ebenso verhält es sich oben, $r \mathrm{~s}$ ist also der Durchmesser des glänzenden Kreises. Das Phantom zeigt, wie unten pag. 19 auseinandergesetzt ist, genau die erwartete Grösse des glänzenden Kreises resp. Ringes. Danach wird also in Figur $\mathrm{I}$ bei der dort angenommenen $\mathrm{Pu}-$ pillengrösse, die reflectirende Zone nicht e $g$, sondern r b sein, denn $h \mathbf{r}$ wird in sich selbst zuräckgeworfen.

Ferner bieten aber auch die Randstrahlen Gelegenheit, die Macula lutea selbst reflectiren za lassen; z. B. wird in Figur II der Randstrahl $g$ f in sich selbst reflectirt und ebenso die, welche von ein wenig höher oder tiefer als $g$ ausgehend, den Punkt $f$ treffen. Ebenso verhält es sich mit $\mathrm{p} \mathrm{r}$. Aber es erhellt auch aus der Figur II, dass, je kleiner die Pupille ist, um so geringer die Zahl der Randstrablen sein wirl, die ron der Macula lutea in die Pupille zurückgeworfen werden. Je kleiner sie ist, um so mehr nähern sich die Randstrahlen dem Parallelismus und den Richtungsstrahlen. Das Phantom zeigt, dass bei mässiger Pupillenweite die Randstrahlen nicht so sehr stören, um nicht eine recht scharfe Grenze des dunkeln Feldes und der glänzenden Umgebung zu zeigen.

So wird sich nun auch die auffallende Erscheinung erklären lassen, dass bei atropinisirter Pupille, wie pag. 3 erwähnt, das Glanzphänomen sehr an Deutlichkeit verliert. Je weiter die Pupille ist und je breiter die homocentrischen Lichtbündel, umsomehr von den schief einfallenden Randstrahlen werden von der Macula lutea 
zur Pupille reflectirt werden, die Contraste und die Grenzen werden verwischt und das ganze Phänomen wird undentlicher. Dazu kommt freilich noch ein anderes Moment: bei grosser Pupille dringt überhaupt viel Licht in das Auge, wird von der Netzhaut zumeist diffus reflectirt, und zwar nicht bloss gegen die Pupille hin, sondern auch gegen andere Theile der innern Häute geworfen und hier zum Theil wieder reflectirt. So entsteht eine merkliche Beleuchtung, gegen welche die zarten Reflexe der Memb. limit. int. für den Beobachter verschwinden. So sieht man letztere ja auch besser in dunkel pigmentirten Augen als bei blonden Individuen. Am deutlichsten erscheint das Phänomen in den Augen brünetter Kinder bei mittlerer Pupillenweite.

Der Pupillendurchmesser ist, wie gesagt, massgebend für die Breite der glänzenden Zone. So ist es auch in der Wirklichkeit, doch sehen wir den Reflex nicht ganz plötzlich aufhören, sondern allmälig vom Rande des gelben Fleckes nach der Peripherie hin abnehmen; abgesehen davon, dass er vielfach auf Grund von darüberhin laufenden Gefässen unterbrochen wird. In Fig. III*) befindet sich das Flammenbildchen des Augenspiegels $19,5 \mathrm{Mm}$. vor dem vordern Brennpunkt $\varphi^{\prime}$ des beobachteten Auges. Es folgt daraus, dass die von einem Punkte a der Flamme ausgehenden Strahlen (die blauen Linien) im Auge convergiren und in der Netzhaut den Zerstreuungskreis $\mathrm{d}$ e bilden. In dem letzteren reflectirt unter anderem der vom Richtungsstrahl $" \varphi^{\prime \prime}$ getroffene Punkt $\varphi^{\prime \prime}$ das Licht diffus (die schwarzen Linien), und die durch die Pupille dringenden Strahlen b f und $\mathrm{c} g$ verlassen letztere parallel und werden von der Sammellinse $1 / 2$ in einem Punkt $P$ vereinigt, zu einem Bilde von

*) Die Figur stellt das reducirte Auge in 2facher Vergrösserung aller Vérhältnisse dar. 
$\varphi^{\prime \prime}$. Daneben wird aber ein Theil der Strahlen, die it. der Richtung $\alpha \varphi^{\prime \prime}$ den Punkt $\varphi^{\prime \prime}$ treffen von der Membr. limit. intern. gespiegelt in der Riehtung des (rothen) Strahles $\varphi^{\prime \prime} h$. Der Letztere wird aber beim Verlassen des Auges, da es sich um ein homocentrisches. Bundel in einem emmetropischen Auge handelt, paraliel deis übrigen vou $\varphi^{\prime \prime}$ ausgehenden Strahlen gebrochen werden und kommt so nach P.

Der Beobachter sieht also in $\mathrm{P}$ die Vereinigung von zwei Strahlengruppen, von denen die eine ein physische. Bild des Netzhantpunktes darstellt, während die ander: uns einen Theil der Beleuchtungsflamme repräsentirt. Beide sind in einem Punkte vereinigt und wemn letzter" einmal das Uebergewicht bekommt, so kann sie das Bilc der anderen ganz verdecken, wie man es bisweilen an Kinderaugen findet. Aehnlich wie uns ein dem Fenster gegenüberhängendes Oelbild durch den Glanz des Firniss verdeckt wird.

Der von einem tiefern Punkte $\beta$ der Flamme $A$ aus. gehende Richtstrah! $\beta \mathrm{m}$ wird nach $\mathrm{mn}$ reflectirt, triff: also die Pupille nicht mehr. Wohl aber könnte ein Randstrahl eines den Punkt $\beta$ benachbarten Bündels den Punkt $m$ in einer solchen Richtung zum Einfallsloti treffen, dass der gespiegelte Strahl die Pupille verlasst. In Figur IV trifft der vom Bündel des tiefer gelegenen Punktes $\gamma$ ausgehende Randstrahl $\gamma \mathrm{s} m$ den Punkt $n$ gerade in der Richtung des Einfallslothes o $\mathrm{m}$, wird so in $m s$ reflectirt und verlässt dann die pupille in de: Richtung $s$ t. Den Punkt $n$ wird also bei diese: Stellung des Flammenbildchens der Beobachternur in Weg: des Randstrables $m \mathrm{~s} t$ glänzen sehen.

Während also die mehr centralen, am Rande der Macula lutea gelegenen Punkte glänzen sowohl vermögu ihrer Richtungsstrahlen als der Randstrahlen benach barter Bündel, reflectiren die mehr peripheren, nahe des. 
Grenze der polaren Zone gelegenen Punkte nur vermöge der Raudstrahlen benachbarter Bündel.

So mag es geschehen, dass die letateren durchweg schwächer glänzen, dass bessere Bedingungen nöthig sind, sie überhaupt glänzen zu lassen, dass sie in späteren Jahren, wo die Durchsichtigkeit und Reflexionsfähigkeit der Membranen continuirlirh nachlässt, ubberhaupt nicht mehr glänzen.

Damit wäre eine Erklärung gegeben, weshalb wir bei Kindern die polare Zone in grosser Ausdehnung glänzen sehen, während wir bei etwas älteren Individuen den Glanz meist nur in nächster Nähe der Macula lutea bemerken. Immerhin ist aber damit der scharfe a us sere Rand des elliptischen glänzenden Reifens, den wir so oft sehen, noch nicht erklärt; denn, um nur das Eine zu erwihnen: wenn derselbe von dem Pupillarrand dircet abhängig wäre, so müssten wir ja einen sphärischen äussern Rand haben, während wir einen elliptischen überall gleich breiten Reif sthen.

Wir müssen uns hier zunächst mit einem andom Glanzphänomen beschäftigten, welches wir an den Gefässen des Augenhintergrundes, und zwar in viel grösserer Excentricität als die polare Zone reicht, bemerken. Fs besteht dies in glänzenden Streifen, die die Arterien begleiten und welche von allen Autoren erwähnt werden und bei Kindern leicht zu sehen sind. Liebreich*) sagt darüber: "Es werden oft die Retinalgefässe von hellglänzenden Streifen begleitet, die bei kleinen Drehungen des Spiegels von einer Seite der Gefässe zur andern überspringen".

Und Schirmer**): "Ich machte die Bemerkung, dass dieser Glanz an der dem Kernlicht des Augur-

*) v. Graefe's Archiv IV. 2, pag. 149.

**) v. Graefe's Archiv X. 2, pag. 149. 
spiegels abgewandten Seite des Gefässes sichtbar wird. Nur selten sieht man auf beiden Seiten der Adern zugleich, wenn dieselben ziemlich in der Mitte des beleuchteten Feldes liegen, diesen glänzenden Streifen".

Dagegen muss ich bemerken, dass ich an Gefässen, wenigstens an solchen, die aus serhalb jener in toto glänzenden polaren Zone liegen, den glänzenden Streifen immer nur an einer Seite des Gefässes und zwar der der Macula lutea abgewandten Seite gesehen habe. Dasselbe meint wohl Schirmer, wenn er sagt: ,ich bemerkte, dass dieser Glanz an der dem Kernlicht des Augenspiegels abgewandten Seite sichtbar wird".

Bekanntlich umgeben zwei von der Papille kommende Gefässe in einem sanften Bogen die Macula lutea. Wenn man zuerst letztere fixirt hat und nun die Augenaxe ein wenig erheben lässt, so dass die obere Netzhanthälfte and in ihr jenes obere horizontale Gefäss in die Gesichtslinie kommt, so sieht man die obere Seite, im umgekehrten Bilde also die untere des Gefässes glänzen. Umgekehrt ist es bei geringer Neigung des Blickes, wo man im umgekehrten Bilde die obere Seite des horizontalen Gefässes glänzen sieht. Ganz entsprechend verhält es sich an vertikalen Gefässen bei seitlicher Blickrichtung, immer glänzt die der Macula lutea abgewandte Seite; und ebenso bei Netzhautgefässen die mehr excentrisch gelegen sind.

Auch diese Erscheinung erklärt sich aus der Niveauverschiebung der Membrana limitans, welche, wenn sie die Retinalgefässe überbrückt, sich von beiden Seiten über die innere Oberfläche der Retina dachförmig erhebt.

Wenn in Figur $\left.V^{*}\right) c$ und d die Stellen jener oben erwähnten Netzhautgefässe bezeichnen, so wird der Strahl

*) Figur V stellt das reducirte Auge nach Listing in doppelter Grösse dar und ist hier als Sagittalschnitt zu denken. 
a s so reflectirt, dass $\mathrm{c} b$ nicht mehr die Pupille trifft; soll er sie treffen, so muss sich die Tangente seines Berührungspunktes $b$ im Sinne von $m \mathrm{n}$ neigen. Dies aber geschieht durch die obere Seite des Daches, in welchem die Membrana limitans das Gefäss c überbrückt. Diese Niveauverschiebung der Membrana limitans durch die Gefässe giebt also den Anlass zu den glänzenden Streifen, welche letztere begleiten, so wie auch nach meiner Meinung zu den ,Lichtbüscheln, welche nach Mautbner*) bisweilen den silbergiänzenden Ring an der Aussenseite krönen" und zu den Zeichnungen, welche nach Schir$\left.\operatorname{mer}{ }^{* *}\right)$ „feinverästelten, hellglänzenden Eisblumen der Fensterscheibe ähneln".

Ich hatte Gelegenheit, diese Verhältnisse an den Gefässen in dem Auge eines Mulattenknaben unseres Waisenhauses zu studiren. Hier, wo das dunkle Pigment der Tapetschicht fast gar kein Licht von der Choriocapillaris durchlässt, wo der Augenhintergrund mehr grau als roth aussieht, treten die zarten Reflexerscheinungen der Retina viel dentlicher hervor als in dem germanischen Auge, wo das rothe Licht der Chorioidea alles andere vernichtet. Hier sah man, wie der Reflex an den Gefässen an einzelnen Stellen breiter war, als an andern, weil die Limitans interna, um das Gefäss zu überbrücken, schon früher die Ebene der Retina verlassen hatte uud so nach Art der Falten wellige und geflammte Linien entstanden.

Uebrigens hat Loring***) einen ähnlichen Gedanken ausgeführt, der mir zuerst aus den Referaten in Nagel's Jahresbericht $\dagger$ ) bekannt wurde. Aber ich

*) loc, cit. 314 .

**) loc. cit. 149.

***) E. G. Loring. Halo round macula Transact. Aner. ophth. pag. $73-81$.

f) loc. cit. pag. 160 und 191. 
halte die Erörterungen für physikalisch nicht ganz richtig. Er sagt, auf die bekannte Zeichnung Max Schulze's*), die den Durchschnitt der Macula lutea darstellt, weisend, folgendes: „Die Gegend, welche in der Zeichnung dargestellt ist, hat in ihrer Form eine entschiedene Aehnlichkeit mit einem flachen Becher dessen land durch eine convexe und dessen Grund durch eine concave Oberfläche dargestellt wird. Wenn wir auf diese gekrümmten Oberflächen als Spiegel blicken, so würden sie Beide ihre Brennpunkte haben, deren einer hinter dem andern liegt, je nach ihrem Krümmungsgrad. Wenn nun Licht vertical auf diese Combination von gakrümmten Ilächen geworfen wird, so würde die Kante des äusseren Randes oder der convexen Oberfläche nach bekannten optischen Gesetzen erleuchtet erscheinen, während die innere oder concave Fläche mebr weniger im Schatten urscheinen würde. So also würden wir das Bild eines dunkeln Centrums, umgeben von einem beleuchteten Rande haben".

Loring scheint $z a$ glauben und in der Zeichnung Max Schultze's zu sehen, dass die Retina, bevor sie sich zu der Macula lutea verdünnte, erst noch eine Ver. dickung erführe, so dass also ein erhabener Reif, ein Wall die Macula lutea umgebe und bei den Experimenten, die er ausgeführt hat, wird allerdings solch ein Wall provocirt. Er hat auf das Loch eines metallischen Augenspiegels ein rundliches Eisen gesetzt und durch cinen Schlag auf dasselbe eine zarte Nertiefung in der Umgebung der Perforation hervorgebracht. Durch Ansetzen einer vorn offenen Holzkapsel an den Spiegel hat er die Camera obscura vollendet (also ohne eine genaue sphärische Krümmung, ohne ein emmetropisches System von Linse und Hornhaut) und hat jetzt das Phantom

*) Archir für mikroskopische Anatomie. 'Tafel VI, Figur 1. 
ophthalmoseopirt und so den glänzènden Reif in der Umgebung des Loches gefunden. Es lässt sich aber leicht srweisen, dass, wenn man irgend ein Metall mit einem sundlichen oder conischen Stempel eindrückt oder perforirt, ohne dass ein Kern wirklich herausgetrieben wird, us dann auf beiden Oberflächen deatlich hervorquillt. is muss ja als dehnbarer Körper zur Seite weichen und so allerdings einen sichtbaren Wall bilden, dessen Scheitel senkrecht auffallendes Licht reflectirt, während die Seitenwände es seitlich ablenken.

Ganz dasselbe gilt von einem anderen Experiment, wo er ein Staniolblättchen mit einer Nadel durchstach, uin zweites gegen die feine Oeffnung andrückte und an diesem in ähnlicher Weise wie zuerst, den glänzenden Ring ophthalmoscopisch betrachtete.

Solch ein Wall entsteht aber nicht bei dem Durchschnitt zweier ungleicher Kugeloberflächen und auch dann nicht, wenn die Kanten abgerundet sind. Die Richtungsstrahlen treffen an den Wänden des kleinen Kugelabschnittes (der Macula lutea oder Fovea centralis) immer auf so geneigte Flächen, dass sie nach innen abgelenlit, nicht zur Pupille zurückgeworfen werden. Auch zeigt «ie Zeichnung Max Schulze's, die er selbst abbildet, diesen Wall nicht. Loring hat nicht berücksichtigt, lass es ankommt auf die Lage der stärkeren Krümmung im hinteren Pol eines sphärischen Apparates mit brechenden Flächen, deren Knotenpunkt vor dem Mittelpunkt der Kugel liegt.

Denken wir uns aber den Uebergang der beiden Kugeloberflächen ineinander nicht plötzlich, sondern allmälig, wie es ja wahrscheinlich der Fall ist, so kann der Durchschnitt derselben an dieser Stelle eine sanft gebogene Linie darstellen, deren Convexität nach vorn und innen gerichtet ist.

Wenn nun die Richtungsstrahlen des Augenspiegels 
nicht in der Axe einfallen, sondern 7. B. bei etwas erhobener Augenaxe den obern Rand der Macula lutea treffen, so fallen sie hier allerdings auf eine leicht convexe Fläche, die je nach ihrer stärkeren odér geringeren Beugung einen bruiteren oder schmäleren streifenartigen Reflex des Augenspiegels giebt und zwar würde er in diesem Falle in umgekehrten Bilde am unteren Rande der Macula lutea erscheinen. Derselbe kann aber wohlgemerkt immer nur böchstens einen Quadranten des Kreises einnehmen, nie auch nur annähernd das Phänomen zur Hälfte oder in toto darstellen und ebensowenig den flächenhaften Glanz der polaren Zone erklären*).

Fine pathologische Beobachtung, die ich machte, bestärkte bei mir die oben entwickelte Theorie. Im Frühling 1872 wurde mir ein 10jähriges Mädchen vorgestellt, die vor 3 Tagen an Neyritis fulminans innerbalb einer Viertelstunde völlig erblindet war. Die ophthalmoscopische Untersuchung zeigte eine leichte Schwellung der Papillen; mir aber fiel besonders an einer derselben auf, dass die innere von der Macula lutea abgewandte Seite dieses Hügels eine breite glänzende Zone zeigte, die je nach der Haltung des Spiegels von der Spitze bis zur Basis des Kegels wanderte. Mit der Heilung des Prozesses schwand das Phänomen mehr und mehr.

Die Erklärung ist nach Obigem einfach: Der Strahl a b (Fig. II), der einen Punkt der normalen Papille trifft, wird nach b c reflectirt und erreicht die Pupille nicht; soll er diese treffen, so müsste sich die Tangente des Punktes $b$ in dem Sinne ron $m \mathrm{n}$ bewegen, was augen-

*) Uebrigens macht Loring 1. c. auch eine Bemerkung über die glänzenden Streifen an den Gefässen, die er auch auf Niveauverschiebung bezieht, ohne jedoch den Vorgang näher zu erläutern. 
scheinlich an der innern Seite des Papillarberges geschieht.

Dass diese Erscheinung nicht öfter beobachtet ist, mag darin liegen, dass Neyritis mit merklicher Schwellung bei Kindern selten ist und bei Erwachsenen wieder die Membranen und brechenden Medien viel von ihrer Durchsichtigkeit und ihrem Glanz verloren haben.

Und noch eine zweite, mir nicht weniger interessante pathologische Bcobachtung möchte ich erwähnen:

Im Frühling 1868 behandelte ich ein 11jähriges anämisches Mädchen, die in einer Nacht an Neyritis retrobulbaris des rechten Auges erblindet war. Das ophthalmoskopische Bild glich, abgesehen von den noch auf Druck pulsirenden und nicht haardünnen Arterien, ganz dem der Embolia centralis Retinae. Das Sehvermögen stellte sich nicht wieder her und nach einem Jahre zeigte sie ganz das Bild der centralen Atrophie. Ich sah die Patientin noch öfter und bemerkte zuerst im Fräbjahr 1873, dass, während links die elliptische dunkle Scheibe mit umgebendem Glanze sehr schön sichtbar erschien, auf dem rechten Auge wohl die Fovea centralis sich ganz deutlich markirte, von dem glänzenden Ringe aber keine Spur zu sehen war, sondern ein matter Glanz ohne alle Differenzirung über die ganze polare Gegend bis an den Rand der Fovea centralis glitt. Gerade so sah ich es auch noch im März 1874.

Wir sehen also hier die Erscheinung - einen sphärischen, im Hintergrunde des Auges gelegenen, in seiner Grösse von dem Durchmesser der Pupille abhängenden, nur von der Fovea centralis unterbrochenen Reflex -, wie wir sie immer im normalen Auge haben müssten, wenn nicht die Einbiegung an Stelle der Macula lutea vorhanden wäre.

Wenn es nun, wie ich glaube, feststeht, dass Liebreich's Theorie nicht hinreicht, so bleibt uns nur folจ. Graefo"s Archiv ftir Ophthalmologie, XXI. 2. 
gende einfache Erklärung: Bei der Atrophie aus extraocularen Ursachen schwindet mit dem Nervus opticus bekanntlich nur die Nervenfaser- und die Ganglienschicht der Retina. Wenn aber die Faserschicht schwindet, so schwindet auch die centrale Depression ihrer innern Oberfläche und damit das besprochene Phänomen. Dagegen bleibt natürlich das Bild der Fovea centralis intact, weil sie in alle übrigen Retinalschichten ausser der Stäbchenschicht hineinreicht.

Um die Theorie zu prüfen, liess ich folgendes künstliche Auge anfertigen: Es wurde eine Glasschale mit dem Radius $1^{\prime \prime}$ geschliffen mit matter äusserer Oberfläche. In dem Pol der inneren Oberfläche liess ich eine mög. lichst kleine Delle schleifen mit $7 / 8$ " Radius. Die Delle zeigte einen Durchmesser von $6 \mathrm{Mm}$; und es berechnet sich ihre Tiefe im Mittelpunkt auf 0,0249 $\mathrm{Mm}$. In ihrer. Mitte wurde noch eine zweite stark concave sphärische Vertiefung von 1,2 Mm. angebracht, um die Fovea centralis darzustellen. Die Scheibe wurde hinten mit rothen Velourpapier überdeckt und vorn durch eine angesetzte Messingschale zu einer Kugel vervollständigt. Vorn wurde in dieselbe als Hornhaut eine sphärische Glasscheibe mit dem Radius $20 \mathrm{Mm}$. eingesetzt (entsprechend den Verhältnissen des menschlichen Auges und ganz gleich denen der Figúr II a). Die Hornhautaxe aber wurde um $6^{0}$ nach Aussen gewandt, wodurch zugleich die sehr störenden Spiegelbilder beim Ophthalmoskopiren gemildert werden. - $10 \mathrm{Mm}$. hinter der Hornhaut befindet sich ein Metallschirm als Iris mit einer kreisförmigen Perforation von $10 \mathrm{Mm}$. und einer Vorrichtung, mittels der man eine Pupille von 7 und $5 \mathrm{Mm}$. einschalten kann. - Hinter dieser, die Stelle des hintern Knotenpunktes $(18,5 \mathrm{Mm}$. hinter der Hornhaut) einnehmend, befindet sich eine Glaslinse von $1 \frac{1}{4} "$ Brenn. 
weite. Die vordere Kammer sowie der Glaskörperraum wurden mit Wasser gefüllt.

Ophthalmoscopirt man jetzt das Präparat (siehe Figur VII) ${ }^{*}$ ) so sieht man in der polaren Gegend eine völlig dunkele, matte kreisrunde Scheibe von etwa $12 \mathrm{Mm}$. Durchmesser mit haarscharfen Rändern. Diese umgiebt eine intensiv glänzende Zone, welche bei der Pupille von $7^{\prime \prime}$ Durchmesser eine Breite von etwa $4,5 \mathrm{Mm}$. hat und deren äussere Begrenzung allmälig aber doch mit bestimmbarer Grenze in den erleuchteten aber nicht glänzenden Augenhintergrund übergeht.

Um den Durchmesser des glänzenden Kreises resp. Ringes festzustellen und ihn mit rs Figur II a, pag. 3 zu vergleichen, verglich ich ihn mit der dunkeln Scheibe, deren wirklicher Durchmesser uns ja bekannt ist, nämlich $6 \mathrm{Mm}$. Ich fand die Breite des glänzenden Ringes gerade so gross, wie die Entfernung vom Rande der Macula lutea zu dem Rande Fovea centralis. Der Halbmesser der Macula latea ist 3,0, der der Fovea centralis 0,6; ihre Differenz 2,4 stellt also jene Entfernung, also auch die Breite des glänzenden Ringes dar. Wenn man sie verdoppelt und zu dem Durchmesser der Macula lutea $6,0 \mathrm{Mm}$. addirt, so bekommt man den Durchmesser der glänzenden Zone selbst, also 10,8 Mm. Vergleicht man diesen Werth mit $r$ in Fig. IIa, so findet man, dass sie ganz übereinstimmen: $r \mathrm{~s}$ ist grösser als 10,5 und kleiner als 11,0 .

Bei Benutzung der kleinen Pupille (5 Mm.) ist die glänzende Zone nur etwa $3 \mathrm{Mm}$. breit, bei der grösseren

*) Die Figur ist nach dem grossen Li ebreich'schen Ophthalmoscop gezeichnet, da es der vielen Reflexe wegen nicht leicht ist mit dem gewöhnlichen Augenspiegel ein reines Bild zu erhalten. Hat man mit jenem Instrument einmal eine gute Einstellung gefunden, so kann man damit das Phänomen sehr bequem beobachten und demonstriren. 
(10 Mm.) um vieles breiter, so dass das Flammenbildchen des Augenspiegels sie nur sehr partiell ausfült und dieZone als solche nur durch viele Drehungen des Spiegels nach einander zum Bewusstsein kommt. Genau im Centrum der dunkelen Scheibe sieht man bei scharfer Einstellung einen leuchtenden Punkt, das physische Flammenbildchen der Fovea centralis. In dessen Umgebung, an den Rändern der Fovea centralis bemerkt man, aber nur bei etwas schiefem Einfallen des Lichtes noch hier und da Abschnitte eines kleineren glänzenden Reifens (Figur VII oben links)*). Er rührt her von dem inneren (centraleu) Rande der Delle an der Grenze der Fovea centralis, von den Punkten $q$ und $r$, Fig. VI. Beobachtet man irgend eine andere, wehr periphere Gegend des Augenhintergrundes, so sieht man nur die mattrothe Zeichnung des Glasschliffes, aber keine spur eines Reflexes. Man sieht also hier an den betreffenden Stellen sehr deutlich, wie sich die glänzende Zone mit einer Kreislinie gegen den nicht spiegelnden aber noch erleuchteten Augenhintergrund absetzt. Diese Kreislinie ist, wie oben erwähut, dus Bild der Pupille, nicht etwa das des runden Augenspiegels, denn wenn man statt des gewöhnlichen Ophthalmoscopes sich eines quadratischen mit quadratischer Durchbohrung bedient, so erhält man ganz dasselbe Bild, nimmt man aber eine quadratische Pupille, so erhält auch die glänzende Fläche eine quadratische Form.

Der Glanz in dem Phantom ist erstens intensiver als der des Auges. Dies rührt meines Lrachtens daher, dass wir es in dem Präparat mit mathematisch sphärischen Oberflächen zu thun haben, während im Auge die Fläche der Membrana limitans interna fort und fort durch die

*) Dieser zweite Reflex ist in der Figur, obgleich er bei den meisten Einstellungen völlig fehlt, der Follständigkeit wegen mit angegeben. 
Erhebung der Retinalgefässe unterbrochen wird. Ferner: die Begrenzungslinie der dunkeln Scheibe ist unvergleichlich schärfer am Phantom, als sie es in der Retina ist, und es durfte dies auch nicht anders sein. Der Durchmesser der Delle ist nämlich ziemlich $6 \mathrm{Mm}$. und ihre Tiefe (wenn man die Fovea centralis ganz unberücksichtigt lässt) $0,0249 \mathrm{Mm}$. Die Macula lutea des menschlichen Auges hat dagegen nur etwa $2 \mathrm{Mm}$. Durchmesser. Es würde danach dasselbe Verhältniss der Radien beider Kugeln 7:8 und diese selbst nach den Grössenverhältnissen des menschlichen Auges angenommen die Tiefe der Delle nur 0,0095 Mm. betragen. Nun werden aber mit der abnehmenden Tiefe der Delle die beiden Curven sich immer mehr dem Parallelismus nähern, mithin der Winkel der Tangenten an der Durchschnittsstelle beider Kugeln (am Rande der Macula lutea), das ist der Neigungsunterschied beider Flächen daselbst, ein viel geringerer sein als im Phantom.

Wenn man aber auch dieselben zarten Verhältnisse in dem Schliff darzustellen vermöchte, so würde, wenn ich die Deutlichkeit der ophthalmoskopischen Bilder des natürlichen und des küustlichen Auges mit einander vergleiche, das Phänomen in letzterem noch immer viel zu deutlich sein. Somit aber kämen wir auf eine so geringe Tiefe der Delle, dass es erklärlich ist, dass eine Verdünnung der Retina an dieser Stelle anatomisch bisher nicht nachgewiesen ist resp. nicht nachweisbar ist. Auch sagt Max Schultze*) „Die Netzhaut ist an der Stelle des gelben Fleckes weichér und zu Luichenveränderungen geneigter; und wahrscheinlich durch die leichtere Quellbarkeit erklärt es sich, dass sich, meist sehr bald nach

*) Stricker, Gewebelehre. Die Retina von M. Schultze, p. 1021. 
dem Tode, diese Stelle als sogenannte Plica centralis aufwulstet."

Uebrigens bilden auch die beiden Flächen gewiss nicht eine scharfe Kante, sondern gehen in einem sanften Bogen in einander über, entsprechend dem ganz allmäligen Aufhören der Nervenfaserschicht in der Macula lutea.

Der den centralen Lichtpunkt bei etwas schief auffallendem Licht bisweilen umgebende helle Kreisabschnitt ist der Reflex der centraleren Theile der Macula lutea. am Rande der Fovea centralis, welche hier wieder annähernd rechtwinklig zu ihren Rǐthtungsstrahlen liegen. Wenn er im menschlichen Auge nicht zu bemerken ist, so könnte es einfach darin seinen Grund haben, dass beide Vertiefungen nicht wie zwei Kugeloberflächen in einander übergehen, sondern in andere Curven ${ }^{*}$ ). Er würde z. B. fehlen, wenn die Macula lutea geformt wäre wie der Mantel eines abgestumpften Kegels. Allerdings würde dann wieder die eine Hälfte der Macula lutea glänzen, wenn man das Auge sich ein wenig seitlich richten liesse, weil dann die Strahlen vom Knotenpunkt aus sie wieder ziemlich rechtwinklig träfen; und zwar beim Blick nach innen die äussere Hälfte im umgekehrten Bilde, beim Blick nach aussen die innere. Dies Verhalten aber habe ich öfter bemerkt; es zieht sich bisweilen, wenn eben der Spiegel nicht gerade auf den hintern Pol gerichtet ist, der Silberglanz über eine Hälfte der Macula hinweg, während die andere dunkel bleibt, wir achten nur nicht darauf, weil wir froh sind, wenn wir des Phänomens in seiner ganzen Ausdehnung habhaft geworden sind. Uebrigens habe ich in der That diesen zweiten concentrischen, kleineren glänzenden Ring

*) Nach Henle (Anatomie II, pag. 662) ist die Fovea centralis eine trichterförmige Grube mit sanft geneigten Wänden. 
öfter im Auge gesehen und neulich bei einem 15jährigen Mädchen fast gerade so deutlich wie den grössern in der Umgebung der Macula Intea. Ein Irrthum war hier nicht möglich, beide Ringe waren âusserst deutlich und der kleinere wurde je nach den Bewegungen des Spiegels auf der einen Seite breiter, auf der andern schwächer und trat hervor, wenn die Augenaxe und der Lichtkegel einen kleinen Winkel mit einander bildeten. Die Pupille war in diesem Auge ziemlich gross. Das ganze Bild glich so dem im. Phantom völlig und war nur etwas weniger intensiv leuchtend.

Das Erscheinen dieses zweiten kleineren Ringes im Phantom machte mich Anfangs an meiner Theorie irre; nachher aber fand ich, durch das Modell aufmerksam gemacht, dásselbe Phänomen auch am menschlichen Auge, das auffallender Weise bisher Niemand erwähnt hat. Der leuchtende Punkt im Centrum der dunkeln Scheibe, das physische Flammenbildehen, entspricht ganz an Entstehung;, Gestait und Ort dem centralen lichten Punkt oder Stern, der in Kinderaugen häufig ist.

Auch steht er nicht immer ganz central, sondern wechselt wie dieser, je nach dem Lichteinfall, in etwas den Ort.

Von den Autoren wird in der Umgebung dieses Lichtpunktes im Bereich der Fovea centralis eine rostbraune oder dunkelrothe Färbung beschrieben. Liebreich erklärt dieselbe aus der (anatomisch) gelben Färbung der Macula lutea and Schweigger daraus, dass an dieser Stelle die Retina sehr dünn ist und deshalb den Farbenton der Chorioidea weniger abdämpft als an allen übrigen Stellen. - Mauthner*) bemerkt gegen die erste Ansicht, dass ja dann der ganze Bereich des gelben Flecks in dieser Farbennuance erscheinen müsste;

*) Lehrbuch der Ophthalmoskopie, pag. 316 . 
und auch gegen die zweite Anschauung, dass die Netzhaut in der ganzen Ausdehnung der Macula lutea dïnn genug sei, um die Farbe der Chorioidea nicht abzuschwächen.

Wenn nun, wie ich glaube, die Oberfläche der Macula lutea auch in etwas zur Papille reflectirt - die Wände der Forea centralis thuen es sicher nicht, weil sie so stark concav sind, dass alle gespiegelten Strahlen zur Bildung des physischen Flammenbildchens verwendet werden, keiner zur Pupille zurückkehrt. So sehen wir, da die Netzhaut ganz durchsichtig ist, hier nur das rothe oder rothbraune Licht der Chorioidea, ohne an seiner Perception durch Netzhautreflexe wie in der Nachbarschaft gestört zu sein, und deshalb erscheint uns der Farbenton an Stelle der Netzhautgrube int ensiver. So sieht man auch am Phantombilde (siehe Figur VII) die Gegend der Fovea centralis dunkler roth, als die übrige Macula lutea.

Etwas, was das Phantom nicht zeigt noch zeigen kann, ist die scharfe äussere (elliptische) Begrenzung des Glanzes, welche wir in manchen Fällen im menschlichen Auge sehen und welche den Reflex als einen schmalen Reifen erscheinen lässt, ganz abgesehen davon, ob der Glanz weiter nach der Peripherie zu wieder erscheint oder nicht. Wir sehen nur im Phantombilde einen etwa $3 \mathrm{Mm}$. breiten und nur allmälig schwindenden glänzenden Ring. Ich glaube, dass diese scharfe Begrenzung in den meisten Fällen durch 2 ganz teine Gefässchen gebildet wird, die die Macula lutea in nächster Nähe umkreisen und radienförmige Aeste in sie hinein senden.

Ein Blick auf die Figur $V$ zeigt, dass die Membrana limitans, welche Gefässe, die in jener Gegend liegen, überbrückt, an ihrer innern, der Macula lutea zugekehrten Seite nicht glänzen kann und so entsteht 
die Unterbrechung des Reflexes, der gleich daneben wieder auftreten kann, noch des öftern in gleicher Weise von Gefässen unterbrochen.

In einem Falle zeigte sich dies mir noch besonders deutlich: hier bildeten die beiden Gefässchen, als sie sich vor der Macula lutea trennten und ehe sie sich hinter ibr wieder genähert hatten, 2 dreieckige Räume (A und B, Figur VIII) and der Glanz, der in toto den elliptischen Reifen zeigte, nahm ausserdem auch diese Dreiecke ein, um dann zu verschwinden und erst gleich hinter den Gefässen wieder aufzutreten. Wo diese Gefässe fehlen und dennoch Theile dieses Reifens sichtbar werden, getrennt von dem mehr peripheren Flächenreflex oder ganz ohne denselben, da könnte man ihn erklären nach Art der pag. 16 erörterten Anschauung des schiefen Auffallens des Lichtbündels auf den nach' vorn innen convexen Rand der Macula lutea.

So kann sich etwa ein Quadrant des glänzenden Reifens bilden, und zwar liegt derselbe dann eigentlich nicht mehr am Rande, sondern im Bereich der Macula lutea. Aber ich habe schon oben mehrmals erwähnt, dass ich auch sonst vielfach den Glanz innerhalb des gelben Flecks habe entstehen sehen. An dem Glasschliff liess sich der allmälige Uebergang der beiden Kugelflächen durchaus nicht darstellen, aber man kann sich eine rohe Vorstellung davon machen, wenn man mit einem scharfkantigen cylindrischen Stempel ein Stückchen aus einem dicken Metallblech herausschlägt. Dabei biegen sich die Ränder etwas um und glänzen, je nach dem Halten der Platte quadrantenweis. In der That glaube ich, dass besonders die letzten Reste des Phä nomens in späteren Jahren so zu erklären sind; aber auch in jüngern Jahren mag dieser partielle Reflex bei schiefem Einfall neben dem totalen bei geradem Lichteinfall oft entstehen. Uebrigens giebt es Fälle, wo beide 
Erklärungen nicht passen und wo ich mir selbst bisher keine Anschauung über die Entstehung der scharfen äusseren Begrenzung der Ellipse habe bilden können.

Und noch in einem Punkte unterscheidet sich das Bild des Phantoms von dem des Auges. An dem erstern sehen wir nämlich auch im aufrechten Bilde etwas von dem glänzenden Reif, wenn auch nur jedesmal kleine Theile und sehr viel lichtschwächer; was bekanntlich am Auge fast nie gelungen ist. Loring erklärt dies negative Resultat der Ophthalmoskopie wohl nicht mit Unrecht daraus, dass das aufrechte Bild stets viel lichtschwächer ist und $z u$ wenig intensiv, um uns den hier sehr viel grösseren Bogen, den wir nur in kleinen Theilen übersehen könnten, zur Anschaunng zu bringen. Ich möchte dem noch hinzufügen, dass, wenn wir überhaupt bei der Untersuchung im aufrechten Bilde bei kleiner Pupille sehr schwer zurecht kommen, wir es noch weniger können, wenn wir dieses ausgedehnte lichtschwache Phänomen erkennen wollen. Wir bedürfen dazu erweiterter Pupillen, dann aber schwinden, wie oben gezeigt, sofort die Glanzphänomen bis zur Unkenntlichkeit.

In dem Phantom, wo wir es mit mathematisch genauen Curven zu thun haben, ist der Glanz gross genug, um auch diese Erscheinung bei mässig weiter Pupille in mässiger Deutlichkeit zu zeigen. 


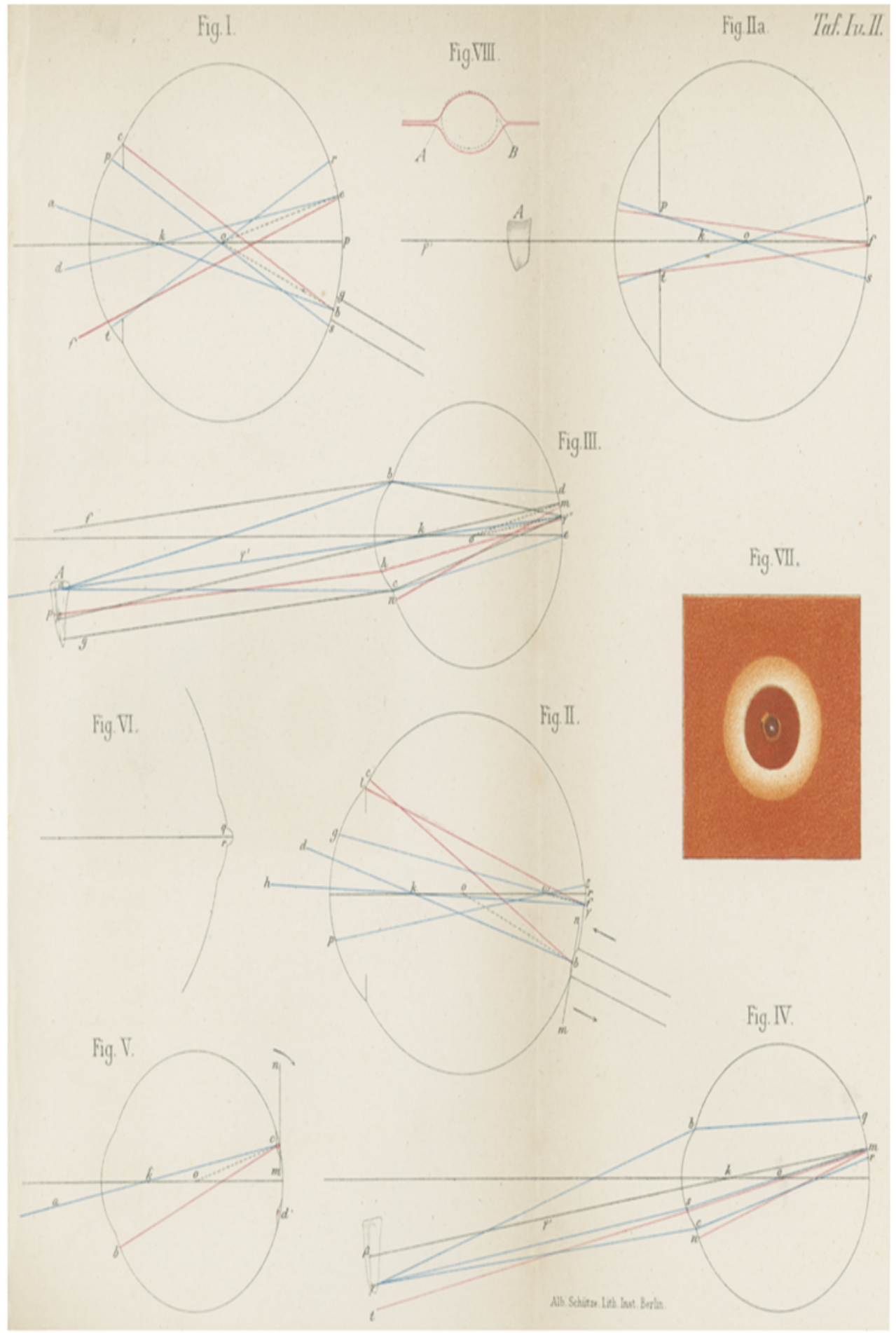

